\title{
Anemia hemolítica causada por Indigofera suffruticosa (Leg. Papilionoideae) em bovinos ${ }^{1}$
}

\author{
José Diomedes Barbosa Neto², Carlos Magno Chaves Oliveira ${ }^{3}$, Paulo Vargas \\ Peixoto $^{4}$, Imke Barbara Pfeifer Barbosa ${ }^{2}$, Sandra Cristina Ávila ${ }^{2}$ e Carlos Hubinger \\ Tokarnia ${ }^{4}$
}

\begin{abstract}
Barbosa Neto J.D., Oliveira C.M.C., Peixoto P.V., Barbosa I.B.P., Ávila S.C. \& Tokarnia C.H. 2001. [Hemolytic anemia caused by Indigofera suffruticosa (Leg. Papilionoideae) in cattle] Anemia hemolítica causada por Indigofera suffruticosa (Leg. Papilionoideae) em bovinos. Pesquisa Veterinária Brasileira 21(1):18-22. Central de Diagnóstico Veterinário, Universidade Federal do Pará, Castanhal, Pará 68740-080, Brazil.

The aereal parts of Indigofera suffruticosa Mill. (family Leg. Papilionoideae) were force-fed fresh to 6 bovines in daily doses of 10 to $40 \mathrm{~g} / \mathrm{kg}$. Cattle breeders of various parts of the Northeast of Brazil accuse this plant as the cause of a non-fatal disease characterized by hemoglobinuria. The disease occurs only in years when the plant proliferates well invading the native pastures. All experimental animals had hemoglobinuria, which was transitory, inspite continuation of the administration of the plant. Two animals had no further manifestations, a third animal showed only slight other manifestations, and the other three had additional symptoms of moderate intensity. These were apathy, whitish visible mucous membranes, rough hair coat, anorexia, descrease in frequency and intensity of the ruminal movements, tachycardia, positive venous pulse and dispnoea. Before the occurrence of the hemolytic crisis the urine had a bluish-green colour. None of the experimental animals died, but one was euthanized whilst showing hemoglobinuria. Post-mortem findings were anemia, the bladder containing wine-red urine, swollen dark-brown kidneys, liver on the outside and on the cutsurface bluish and with perceptible lobular design. The main histological changes were found in liver and kidney. In the liver there was coagulative necrosis and cloudy swelling and/or cytoplasmatic microvacuolization of the hepatocytes; in the kidney there was severe nephrosis, associated with large amounts of filtrate and/or hemoglobine in the Bowman spaces, in the tubules and also in the cytoplasm of the epithelial cells.
\end{abstract}

INDEX TERMS: Poisonous plants, plant poisoning, Indigofera suffruticosa, cattle, pathology, hemolytic anemia.

RESUMO.- As partes aéreas de Indigofera suffruticosa Mill. (família Leg. Papilionoideae), planta incriminada pelos criadores de diversas áreas do Nordeste por doença caracterizada por hemoglobinúria em bovinos, foram administradas por

\footnotetext{
${ }^{1}$ Aceito para publicação em 22 de janeiro de 2001.

${ }^{2}$ Central de Diagnóstico Veterinário (CEDIVET), Universidade Federal do Pará (UFPA), Rua Maximino Porpino da Silva 1000, Bairro Centro, Castanhal, Pará 68740-080.

${ }^{3}$ Médico Veterinário, aluno de Mestrado em Ciência Animal, Centro Agropecuário, UFPA.

${ }^{4}$ Depto Nutrição Animal e Pastagem, Universidade Federal Rural do Rio de Janeiro (UFRRJ), Km 47, Seropédica, RJ 23851-970; bolsistas do CNPq (305010/ 76-VT e 302342/86-9).
}

via oral a seis bovinos, em doses diárias repetidas de 10 a 40 $\mathrm{g} / \mathrm{kg}$, Todos os animais experimentais apresentaram hemoglobinúria, porém passageira, apesar continuidade da administração da planta. Dois desses bovinos não apresentaram manifestações adicionais, um terceiro animal evidenciou manifestações leves, e os três outros, sintomas adicionais de intensidade moderada: apatia, mucosas visíveis de coloração esbranquiçada, pêlos arrepiados, anorexia, diminuição da frequiência e intensidade dos movimentos ruminais, taquicardia, pulso venoso positivo e dispnéia. Antes da crise hemolítica a urina apresentava coloração verde azulada. Nenhum animal experimental morreu, porém um foi sacrificado durante a fase hemoglobinúrica. À necropsia observaram-se anemia, bexiga contendo urina cor de vinho tinto, rins aumentados de volu- 
me com coloração marrom-escura, fígado, na superfície e ao corte, de coloração azulada com lobulação perceptível. As principais alterações histológicas foram verificadas no fígado, sob forma de necrose coagulativa e tumefação e/ou microvacuolização citoplasmática dos hepatócitos, e no rim representadas por acentuada nefrose, associada a grande quantidade de filtrado e/ou hemoglobina nos espaços de Bowman dentro de túbulos e do citoplasma das células epiteliais.

TERMO DE INDEXAÇÃO: Plantas tóxicas, intoxicação por planta, Indigofera suffruticosa, bovino, patologia, anemia hemolítica.

\section{INTRODUÇÃO}

Em 1997, na região do Vale do Jaguaribe, Ceará, obtivemos históricos de uma enfermidade de etiologia obscura que vinha ocorrendo há diversos anos e que cursava com hemólise, hemoglobinúria, anemia, abortos e que vinha afetando bovinos. De acordo com os dados fornecidos pelos criadores desta região, a doença só ocorre em determinadas pastagens onde grassa uma planta conhecida como "anil" ou "anileira". Trata-se de uma planta invasora bem adaptada às condições do Nordeste do Brasil que, em anos de boa pluviosidade, aparece em quantidade suficiente para provocar surtos de intoxicação. Os bovinos apresentam os primeiros sintomas poucos dias após serem colocados nos pastos onde ocorre a planta. A morbidade oscila em torno de $50 \%$ dos animais, sem distinção de sexo ou idade. A retirada dos animais dessas pastagens, no início do quadro clínico, possibilitaria rápida recuperação.

Segundo os dados obtidos nas regiões visitadas, o prejuízo econômico está relacionado com a perda de peso e queda na produção de leite dos animais acometidos, assim como com o tratamento realizado para outras enfermidades que cursam com hemoglobinúria. Muitos criadores mencionaram que alguns animais abortam, fato esse ainda não confirmado por nós. Animais já muito anêmicos e fracos, geralmente não acompanham mais o rebanho e muitos deles terminam morrendo.

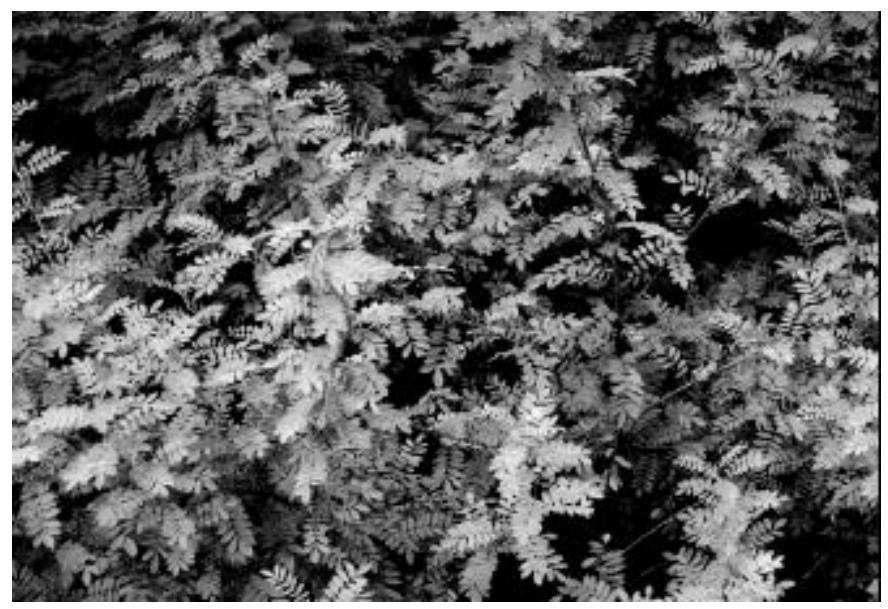

Fig. 1. Indigofera suffruticosa Mill. (fam. Papilionoideae).
Encontramos grandes quantidades da planta, mais tarde identificada como Indigofera suffruticosa, também em outras regiões dos Estados do Ceará, de Pernambuco e do Rio Grande Norte, sempre com o histórico da ocorrência da mesma enfermidade em bovinos. Também no Estado da Paraíba há grande quantidade da mesma planta (Riet-Correa 2000). Vimos pequena quantidade da planta no Estado do Pará, sem históricos de ocorrência da doença.

Na literatura não encontramos dados sobre a toxidez de I. suffruticosa. $\mathrm{O}$ presente trabalho experimental em bovinos foi realizado para comprovar a toxidez da planta, determinar as doses letais e estabelecer o quadro clínico-patológico da intoxicação.

\section{MATERIAL E MÉTODOS}

O estudo foi executado na Fazenda Cajazeiras no município de Jaguaribe, Estado do Ceará.

Foram realizados experimentos com Indigorera suffruticosa Mill. ${ }^{5}$ (Fig. 1 e 2), em um total de seis bovinos clinicamente sadios, mestiços, machos, com idade de aproximadamente 18 meses, pesando 88 a $128 \mathrm{~kg}$. As partes aéreas da planta adulta (com frutos) foram coletadas sempre no mesmo local entre os dias 10 e 20 de maio de 1999 e 17 a 30 de maio de 2000. A administração da planta foi realizada logo após a coleta, manualmente por via oral, em doses diárias de 10, 20, 30 e $40 \mathrm{~g} / \mathrm{kg}$. Durante o ensaio, os animais foram mantidos em estábulo de alvenaria, alimentados com capim Cameron, recebendo água a vontade. $\mathrm{O}$ apetite dos animais foi avaliado oferecendo-se ração concentrada.

Exames clínicos foram efetuados duas vezes ao dia, a partir da administração da planta, avaliando-se e cronometrando-se os seguintes parâmetros: atitude, postura, grau de hidratação, coloração das mucosas, dipsia, temperatura retal, apetite, motilidade ruminal, peristaltismo intestinal, aspecto das fezes, freqüência e alterações cardio-respiratórias.

As avaliações do quadro hematológico, isto é, as determinações do hematócrito, da hemoglobina e a contagem de hemácias, foram 5Planta coletada por nós no Município de Jaguaribe, Ceará, e identificada
pelo Dr. Marcus Nadruz, Jardim Botânico do Rio de Janeiro.

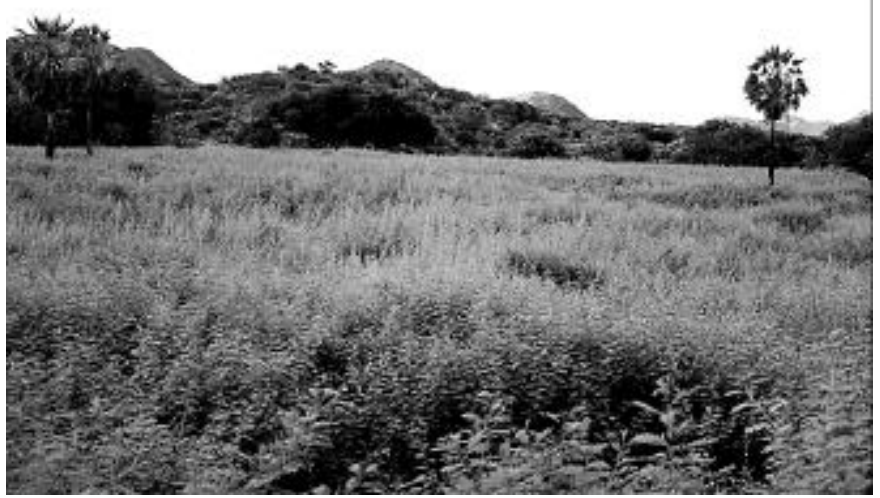

Fig. 2. Pasto infestado por Indigofera suffruticosa, no Vale do Jaguaribe, Ceará. 


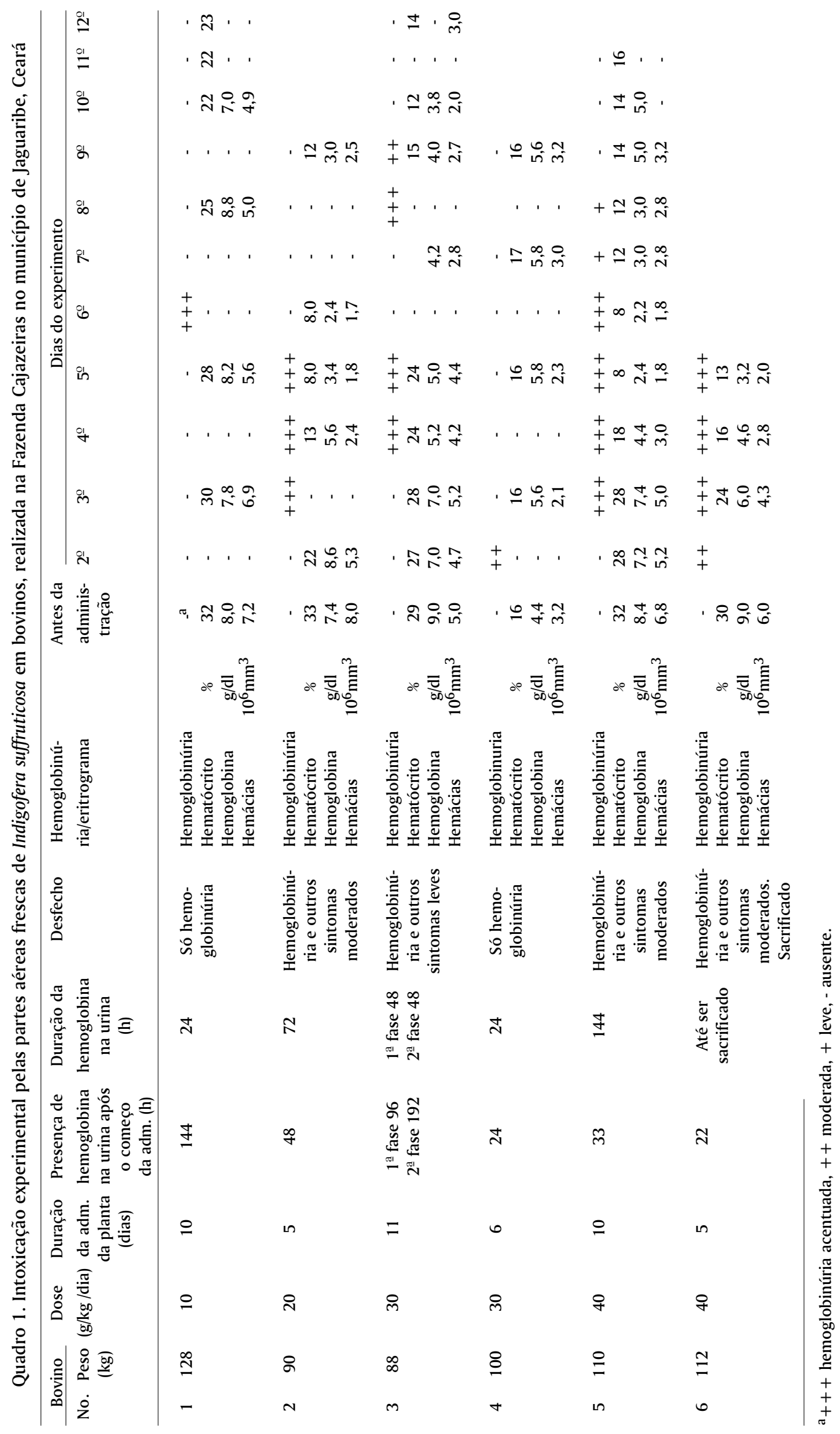


feitas antes do começo do experimento e depois, sempre após a administração da planta, com intervalos variados de acordo com o estado clínico do animal. O sangue foi colhido da veia jugular em tubo de ensaio com capacidade para $10 \mathrm{ml}$ contendo três gotas de EDTA a $10 \%$, utilizando-se agulhas descartáveis 40x16.

O exame da urina, para verificação de hemoglobinúria, foi realizado antes e após a administração da planta, de acordo com a evolução do quadro clínico, sempre com intervalos menores de 12 horas.

\section{RESULTADOS}

Os principais dados dos experimentos em bovinos intoxicados por Indigofera suffruticosa, estão expostos no Quadro 1.

Todos os animais que receberam as doses de $10 \mathrm{a} 40 \mathrm{~g} / \mathrm{kg} /$ dia (Bov. 1, 2, 3, 4, 5 e 6) apresentaram hemoglobinúria, que foi passageira, apesar de os animais continuarem a receber a planta. Dois desses bovinos (Bov. 1 e 4) não apresentaram outros sinais clínicos; um terceiro animal (Bov. 3) evidenciou manifestações adicionais leves, e os três restantes (Bov. 2, 5 e $6)$, sintomas de intensidade moderada.

O quadro clínico observado nos Bovinos 2, 3, 5 e 6, caracterizou-se por hemoglobinúria, apatia, mucosas visíveis (ocular, prepucial e bucal) esbranquiçadas, pêlos arrepiados, anorexia, diminuição de freqüiência e intensidade dos movimentos ruminais, taquicardia intensa (acima de 140 batimentos por minuto) com alteração no ritmo e na intensidade, pulso venoso positivo, vasos episclerais pouco visíveis, aumento na frequiência e na intensidade dos movimentos respiratórios. Evidenciou-se coloração azulada da mucosa prepucial em decorrência do pigmento existente na planta. Após a centrifugação do sangue, o plasma sanguíneo se apresentava hemolisado durante a fase da hemoglobinúria. É importante reportar que sempre antes da crise hemolítica, a urina dos animais intoxicados apresentou uma coloração verde azulada.

Nos animais que receberam $40 \mathrm{~g} / \mathrm{kg} / \mathrm{dia}$, a hemoglobinúria ocorreu rapidamente e era muito duradoura.

Com relação aos achados hematológicos, a queda mais acentuada foi observada a partir do terceiro dia do experimento, principalmente nos animais que receberam doses de $40 \mathrm{~g} / \mathrm{kg}$ dia (Bov. 5 e 6), com exceção do Bovino 2 , que apesar de receber somente $20 \mathrm{~g} / \mathrm{kg} / \mathrm{dia}$ também apresentou uma acentuada queda nos parâmetros sanguíneos.

Os achados de necropsia no Bovino 6, sacrificado durante a fase de hemoglobinúria, foram mucosas pálidas, sangue aquoso, bexiga contendo urina com cor de vinho tinto, rins aumentados de volume com coloração marrom-escura, fígado na superfície e ao corte azulada e lobulação perceptível.

As principais alterações histopatológicas foram observadas no fígado e nos rins. No fígado, os cortes histológicos evidenciam áreas de necrose coagulativa, caracterizada por marcada eosinofilia citoplasmática e figuras de picnose ou cariorrexia, afetando, sobretudo, hepatócitos das zonas centrolobulares. Essas lesões, entretanto, são de distribuição bastante irregular; em alguns lóbulos, a necrose praticamente circunda as veias centro e sublobulares, em outras apresenta-se sob forma de focos adjacentes a esses vasos (necrose paracentral). Em alguns lóbulos, a destruição de hepatócitos se estende até a zona intermediária. Externamente a essa lesão, sob forma de largas faixas, observa-se acentuada tumefação e/ou microvacuolização citoplasmática, acompanhada de leve picnose nuclear, localizada predominantemente nas zonas intermediárias do lóbulo hepático. Em algumas áreas verifica-se apenas a lesão degenerativa, sem a necrose coagulativa. Adicionalmente, há leve a moderada retenção biliar ("trombos biliares”).

Nos rins verifica-se acentuada nefrose caracterizada por tumefação e degeneração, por vezes com evolução para lise, das células epiteliais dos túbulos do córtex e da junção corticomedular, associada a grande quantidade de filtrado glomerular e/ou hemoglobina nos espaços de Bowman, dentro de túbulos (sob forma de glóbulos, grânulos ou cilindros), e também dentro do citoplasma das células epiteliais ("degeneração em gotas hialinas”). Na medular, observa-se grande número de cilindros hialinos e/ou granulares com discreta/leve dilatação dos túbulos.

No rúmen há leve a moderada hiperqueratose paraqueratótica.

\section{DISCUSSÃO}

Os experimentos comprovam que Indigofera suffruticosa é a causa da enfermidade hemolítica não-fatal que acomete bovinos em algumas áreas da Região Nordeste.

No Brasil conhecemos apenas duas outras plantas de interesse pecuária capazes de induzir à hemólise em animais de fazenda: Brachiaria radicans (Andrade et al. 1971a,b) e Ditaxis desertorum (Tokarnia et al. 1997, 2000).

O quadro clínico-patológico na intoxicação por Brachiaria radicans em bovinos, descrito por Vilalobos et al. (1981), é muito semelhante ao aqui descrito. Essa planta, porém, conhecida como "braquiária-do-brejo", não ocorre nas áreas secas do Sertão, onde há I. suffruticosa. Além do mais, a eliminação de urina azulada é suficiente para estabelecer a diferenciação. Também o quadro clínico-patológico da intoxicação por $D$. desertorum, planta responsável por anemia hemolítica em bovinos no Oeste da Bahia, é semelhante ao da intoxicação por Indigofera suffruticosa. Porém enquanto na intoxicação pela última o fígado apresenta coloração azulada, na intoxicação por $D$. desertorum o fígado tem coloração alaranjada

A queda acentuada no hematócrito (queda de até $8 \%$ ), hemoglobina em torno de $2 \mathrm{~g} / \mathrm{dl}$ e número de hemácias abaixo de $2,0 \times 10^{6} / \mathrm{mm}^{3}$ foram verificados na intoxicação por $I$. suffruticosa e também foram encontrados nos animais intoxicados por $D$. desertorum (Tokarnia et al. 1997).

Outro ponto em comum entre as intoxicações por $I$. suffruticosa e $D$. desertorum é a rápida recuperação dos animais observada em ambas enfermidades, apesar de os animais continuarem a receber a planta. Apesar da grande quantidade da planta administrada aos animais na intoxicação por I. suffruticosal, não foi verificado o efeito cáustico produzido por $D$. desertorum administrado em dose elevada. Outra diferença diz respeito à toxidez dessas plantas: foram necessárias doses acima de $10 \mathrm{~g} / \mathrm{kg} /$ dia de $I$. suffruticosa para causar um quadro de hemoglobinúria nos animais, enquanto que em relação a $D$. desertorum, doses diárias de 1 a $2,5 \mathrm{~g} / \mathrm{kg}$ foram 
suficientes. Sob o ponto de vista histopatológico, as lesões foram muito semelhantes, porém enquanto que na intoxicação por $D$. desertorum predominava a necrose, na intoxicação por I. suffruticosa predominava a tumefação e/ou microvacuolização citoplasmática dos hepatócitos.

Agradecimentos.- Ao Conselho Nacional de Desenvolvimento Científico e Tecnológico (CNPq) pela bolsa concedida para realização deste trabalho, aos Proprietários Luciano Ferreira da Rocha e Luiz Gonzaga Barbosa por ter cedido os animais e as instalações para realização do experimento, e ao Dr. Marcus Nadruz, Jardim Botânico do Rio de Janeiro, pela identificação da planta.

\section{REFERÊNCIAS}

Andrade S.O., Peregrino C.J.B. \& Aguiar A.A. 1971a. Estudos sobre Brachiaria sp (Tanner Grass). 1. Efeito nocivo para bovinos. Arqs Inst. Biológico, S.Paulo, 38(3):135-150.

Andrade S. O., Retz l. \& Marmo O. 1971b. Estudos sobre Brachiaria sp. III. Ocorrência de intoxicação de bovino durante um ano (1970-1971) e níveis de nitrato em amostra de gramínea. Arqs Inst. Biológico, S.Paulo, 38(4):239-252.

Riet-Correa F. 2000. Comunicação pessoal (Lab. Diagnóstico, UFPel, Pelotas, RS).

Tokarnia C.H., Chagas B.R., Chagas A.D. \& Silva H.K. 1997. Anemia hemolítica causada por Ditaxis desertorum (Euphorbiaceae) em bovinos. Pesq. Vet. Bras. 17(3/4):112-116.

Tokarnia C.H., Döbereiner J. \& Peixoto P. 2000. Plantas Tóxicas do Brasil. Editora Helianthus, Rio de Janeiro.

Vilalobos S.J., Meneses G.A., Leon C.S. \& Carballo C.G. 1981. Clinica y patologia de la intoxicacion com Brachiaria radicans Napper (Tanner Grass). Cienc. Vet., Costa Rica, 3:163-166. 